\title{
The Effects of Learners' Cognitive Styles and Article Structure Diagram on English Reading
}

\author{
Zhao Rongbin \\ School of Foreign Language, Huaiyin Normal Univarsity, Huai'an, \\ Jiangsu, 710126, China)
}

\begin{abstract}
Based on the characteristics that students can not allocate attentional resources in English reading comprehensively, this study uses the experimental method to study the effect of the article structure diagram on understanding and maintaining various types of information for non-English majors in different cognitive styles.The results indicate that: (1) Signals can effectively improve the student's understanding and reserving text's information. In reading text, the signal effects are evident. (2) There are differences in the understanding and reserving different types' information for readers of different cognitive styles. The text marked by incomplete signals resulted in the increase of the subordinate information for both field-dependent and field-independent readers, and the increase of the structured information for field-dependent readers. The text marked by complete signals resulted in the increase of the structured information for field-dependent readers and the increase of the subordinate informations for field-independent readers. Field-independent readers have a better performance in reserving the structured information in the condition of no signal.

Key Words: cognitive style; information-reserving; article structure diagram
\end{abstract}

\section{First, research background}

Reading as a linguistic activity is a process of constructing a coherent cognitive representation in a hierarchical manner with the aim of extracting the meaning of the text. The reader's understanding of the article depends on the background knowledge, conceptual capabilities and the interaction of processing strategies. Reading comprehension process is the process of interaction between text and 
readers' background knowledge. If the reader lacks the corresponding background knowledge, we need to use the corresponding language mechanism, such as the article mark to infer the logical relationship between the sentences, and then construct the important information of the characterization of the article. [2] Thus, readers to identify and use the signal is an effective way to improve their reading abilities.

Article markers refer to words, phrases, sentences, or special symbols that appear at different points in the article. They do not add new content to the article itself, but emphasize the structure or specific content of the article. [3] The existing studies have shown that the article markers played a certain role on the reading of cognitive processing, information understanding and maintaining, which is the article marking effect. [4-6] However, the current scholars emphasize research methods of the macro structure of the article markers and the specific content of the micro-markers as a marker effect, few people to study the effect by using article structure diagrams. Based on the concept of article marking, the article structure chart will be a more clear and more logical framework of the important information in the text, and it also does not provide additional information, in line with the definition of article markers. It is necessary to study whether it can help readers to recognize the structure of English articles to promote their understanding of the article.

In addition, the article mark effect is also affected by many factors. First, reading material affects the marking effect. Different from reading the native language, English reading needs more information processing time to deal with word cognition and grammatical structure. Second, the article marking effect is also affected by the reader. Because reading is a cognitive process, so the reader's cognitive style is different, it will be the same knowledge from different perspectives for analysis and construction. Cognitive style is the way learners recognize, process, store and extract information in the cognitive process, and the starting point for learners to solve the problem. [7] Witkin pointed out that the socalled "field" is the surrounding environment, its perception of people have different degrees of influence. Field independence tend to use the internal reference signs to take the initiative to external information processing. Based on differences in cognitive styles, Richards and Fajen's research shows that the reader's cognitive style is a factor influencing the effect of article marking. [8] $\mathrm{He}$ Xianyou took junior high school students for the test and found in marked conditions, field independence and field dependency of the results did not differ, but in the case of unmarked field independence is better than field-dependent results. [9] Song Guangwen also took junior high school students as subjects and found that the article mark on different cognitive styles of prose learning has no significant effect, but the mark can promote field dependent students on the study of the text. [10] However, at present for different cognitive styles of non-English majors in English reading in the article structure of the marker effect of research is rarely involved. 


\section{Second, the purpose of the study}

In summary, the author will systematically explore the effects of the subject and object factors on the article marking. The object factor refers to the article structure diagram. The article is divided into three types of marking: unmarked, semi-marked and full mark. The subject factors include field-independent cognitive style and field-dependent cognitive style. In this study, non-English majors are selected as the research object, and the article structure diagram is used as the mark of the article. Compared with the different cognitive styles of learners' English reading comprehension and information retention ability, the following questions are discussed: 1 . Whether it can improve the comprehension and retention ability of the non-English majors' information in English articles; 2. whether there is a certain correlation between the different markings (unmarked, semi-marked and full-marked) affect the retention of the article information and the reader's cognitive style.

\section{Research methods}

1. The subjects of this study were 195 randomly selected students from Xi'an University of Electricity and Technology, including 123 males and 72 females with an average age of 19.6 years.

\section{Experimental Materials}

Reading comprehension test essay. The comprehensive consideration of new words, cultural background and other factors. The author chose an 831 words in the introduction of earthquake prediction English papers were rewritten as unmarked, semi-marked and full tag three version.

Cognitive style measurement tools. Cognitive style measurement tools used "Mosaic Graphics Test" by Beijing Normal University Fu Jen Application Development of Psychological Development Center. Subjects were asked to distinguish nine simple target graphics from three sets of 29 complex graphics.

3. Experimental design

In the experiment, the two factors (cognitive style: field dependency, field independence) $\times 3$ (mark: no mark, semi mark, full mark) were designed to investigate the information retention in English reading.

4. Experimental procedures

(1) Cognitive style measurement

Using collective measurement, all tests are required in 13 minutes in order to complete the three groups of graphics recognition. After the test is finished, the primitive score of the pattern mosaic test is converted into the standard score and compared with the norm. Then, the subjects whose standard score is greater than 55 are determined as the field independence, and the subjects with standard score less than 40 are determined as field dependent. Statistics were deleted when the failed subjects, the final field of independent and field-dependent selection of 90 people. 
(2) English reading test

After clarify field independence and field dependent as two groups, the experiment starts in a group manner. The two groups were equally divided into three different treatments: the subjects receiving the first treatment only saw the original texts that did not provide any redundant information; the subjects who received the second treatment could see the original text and parts The rest of the subjects need to be supplemented according to the content of the article. The subjects receiving the third treatment can see the original text and the complete structure of the article. Subjects were asked to read the article in 10 minutes, and then the article back, the test. The test questions consisted of 10 short answer questions, 2 points for correct answers, and 0 points for incorrect answers, for a total of 20 points. By calculating the pearson coefficient of two scorers, $r=0.812$, $\mathrm{P}<0.004$, reaching a significant level, indicating that the score is credible.

Fourth, the results

First of all, the author makes a two-way analysis of variance of cognitive style (field dependency, field independence) and article mark types (unmarked, semi-marked and full marked) on the total information, the subject information and the detail information respectively. Descriptive statistical results in Table 1 and Table 2.

The statistical results in Table 1 and Table 2 show that, firstly, there is a significant main effect on the retention of total information in the article (see Table $1, \mathrm{~F}=14.131, \mathrm{P}=0.000<0.01$ ). $\mathrm{M}=14.50$ ) and the full-tag (see Table 2, $M=14.18$ ), the recall of the group was higher than that of the untagged group (see Table 2, M = 13.25). However, the main effect of cognitive style was not found (see Table $1, \mathrm{~F}=0.607, \mathrm{P}=0.437>0.05$ ).

Second, in the maintenance of the topic information, the article mark has obvious main effect (see Table $1, \mathrm{~F}=7.271, \mathrm{P}=0.001<0.01$ ). $\mathrm{M}=7.20$ ) and total markers (see Table $2, \mathrm{M}=7.10$ ), the difference was statistically significant $(\mathrm{P}<0.05)$, and the difference was statistically significant $(\mathrm{P}<(\mathrm{F}=0.262, \mathrm{P}=$ $0.61>0.05)$ between the semi-labeled group and the full-labeled group. The cognitive style also had a significant main effect (see Table $1, \mathrm{~F}=6.021, \mathrm{P}=$ $0.015<0.05$ ). Field independent recall (see Table $2, \mathrm{M}=7.13$ ) was statistically significant higher than field-dependent subjects (see Table 2, $\mathrm{M}=6.76$ ). There was a significant interaction between cognition style and article labeling (see Table $1, \mathrm{~F}=5.583, \mathrm{P}=0.004<0.01$ ).

Thirdly, in the maintenance of detail information, the article mark has obvious main effect (see Table $1, \mathrm{~F}=5.801, \mathrm{P}=0.004<0.01$ ). $\mathrm{M}=7.38$ ) and full-label (see Table 2, $\mathrm{M}=7.08$ ) were higher than those in the untagged group (see Table 2, $\mathrm{M}=6.72$ ), while the semi-marker and full-label groups did not Showing a statistically significant difference $(\mathrm{F}=1.522, \mathrm{P}=0.22>0.05)$. The cognitive style also had a significant main effect (see Table $1, \mathrm{~F}=14.234, \mathrm{P}=0.000<0.01$ ). Field-dependent recall (see Table 2, $\mathrm{M}=7.30$ ) was higher than field independence (see Table 2, M = 7.08). In addition, the interaction between the cognitive style and the article marker reached a significant level (see Table 1, F $=$ $3.392, \mathrm{P}=0.036<0.05)$. 


\section{Discussion}

Reading is a complex psychological construction process. The acquisition of reading information is not only closely related to the reader's cognitive style, but also related to the use of the article mark. The statistical results show that the article structure diagram improves the total amount of information of the subjects, but also makes the subjects of different cognitive styles have different influence on the maintenance of various types of information.

1. Effect of the Mark on Maintaining Various Information.

(1) Semi-mark is provided for the part of the article structure diagram the rest of the tests need to be based on the understanding of the article added complete. The statistical results show that the participants can use the structure diagram in the semi-mark to identify the structure of the article and construct the article structure. This characterization also facilitated re-processing of subjects' information, and thus showed a good preservation effect on the subject information.

Full mark offers the whole article organizing charts. So full mark has a lot common with semi mark on the coherence. In the reading process, the complete structure diagram plays the role of structural clues, which helps to form good structural conditions, and finally promotes the maintenance of details about subject and subject. However, in contrast to the semi-marker condition, there is no blank space in the whole marker. Therefore, it is difficult to actively identify and search the content of the space, and the processing and memory are lacking. The recall of information is lower than the semi-mark.

Through the experiment, the clear structure of the article marks the article are conducive to the understanding of the article to form a clear article characterization. This result is consistent with the results of Bai Xuejun's research. Bai Xuejun (2004) took college students for example, with the mark to help students build the article structure, and ultimately to promote their understanding of the content of the article. The semi-mark and the full mark facilitated the subjects to recall more subject information and detailed information. Under the condition of semi-mark, the subjects had the highest scores on all kinds of information, which means that they had the best understanding of the article, followed by the whole mark and the lowest score without marking (Table 2).

(1) In the absence of marking conditions. Field-independent subjects had higher retention of subject information in the unmarked condition. The results are in agreement with the results of Richard (1997) and He Xianyou (2000). This indicates that field-independent persons have less difficulty in determining the structure of complex information arrangements than field-dependent ones, and they are more likely to detect the structure and important content of the article than field dependents. Field independent are good at comprehensive analysis of the method, and can seize the thrust of the problem. They can focus on the content related to the purpose of reading, the better to achieve the goal of reading activities, so they are more likely to get good grades in reading. At present, many studies have found that field independent in the cognitive activities demonstrated monitoring skills, information organization and extraction capabilities metacognitive skills. [11] These skills can help them to correctly read the article 
at all levels of information, efficiently and quickly understanding of the thrust of the article, and to produce a good migration. So field independent subjects can recall more of the subject information, but in the details of the memories did not show an advantage.

(2) Under semi-mark conditions. The results show that semi-markers promote the retention of topic information and detail information in field-dependent subjects, and promote field independence to maintain detail information.

The experimental results show that field dependents are consistent with field independence in the ability to obtain information, but their strategies and points are different, which are consistent with the findings of Song Guangwen's scholars. [10] Field Dependent attention to detail information, non-strategic learners, but they do not lack cognitive skills, but need to prompt it. The semi-markers remind participants of the potential cognitive reorganization skills, promote their attention to the processing of the article, and guide the field-dependent subjects to shift from the case table strategy to the structure strategy, and construct a hierarchical organization and conceptual content. So the semi-mark strengthens the retention of topic information by field dependents, and this result supports Meyer's strategy transformation hypothesis. But for field-independent subjects, the recall of the subject information does not require the guidance of the marker. Regardless of whether the article can reflect the structure of the mark, field independent subjects can detect the structure and important content of the article. The results also show that the field independent subjects tend to be based on the internal reference, on cognitive restructuring ability, and on finding the logical relationship between the article.

(3) Under full-mark conditions. As the full mark of the complete structural map, the mechanism and principle of the field-dependent subject and the fieldindependent subjects are consistent with the semi-labeling conditions, and the full-mark also facilitates the field-dependent subjects to carry out the strategy conversion, and ultimately improve their access to information on the subject and maintain. For field independent subjects, the presence or absence of full marks had no effect on their recall of subject information. As a result, there was no statistical difference in the retention of subject information between fieldindependent subjects and field-dependent subjects.

In the maintenance of detail information, the presence of the full mark facilitates the construction of the subordinate information of the independent subject on the related topic, that is, after a certain subject is accessible, they can easily make this access to the corresponding detail information Of the memories. Therefore, the expansion effect of subjective accessibility in the field of independent subjects who took effect, increasing the details of their information memories.

\section{Conclusion}

In summary, the experimental results show that the article structure is a combination of the article information. If the reader can correctly recognize the 
structure of the article and form a clear structural representation, it will promote their understanding and maintenance of the important content of the article. Meanwhile, the article structure mark provides important clues for the reader to help them form a clear structural representation, and ultimately to improve their reading level. Moreover, the structural markers have different effects on the processing and maintenance of the article information, that is, the labeling effect is different. In the process of reading, the subjects constructed the structural representations of the subject content by using the full mark and the semi mark, and instructed them to recall the subject information. And fill in the blanks in the semi-markers to help the subjects to identify and strengthen the details of the article, to promote their memories of the details of the information.

Cognitive styles affect article marking. The results of the study are as follows: 1. The cognitive style and understanding of the topic and detail information of different subjects are affected by the structure marking. Field independent subjects have a good grasp of the theme structure of the article, this ability is not affected by the article mark. Field-independent subjects' retention of detailed information on the articles is influenced by the markings, and semimarks and full marks can facilitate their recall of detail information. 2. Fielddependent subjects under the conditions of full-mark and semi-mark cues improved the understanding and maintenance of the subject information. At the same time, the blanks in the semi-markers also stimulate the field-specific information in the article identification, construct the representation of the specific information, and use this representation to improve their memories of the details of the information.

The results of this study have some enlightening significance for college English reading teaching in the future. The organization chart as a way to mark the article can effectively improve the non-English majors in the article to understand and maintain the various types of information. However, because students of different cognitive styles have different degrees of dependence on markings and different information, students should be trained according to their different cognitive styles when they are trained in English reading strategies, which will be expected to improve students' ability and efficiency of reading comprehension.

\section{References:}

[1] Coady J A. Psycholinguistic Model of the ESL Reaser [M]. In Ronald Mackay, Barkman \& R.R. Jordan (eds). Reading in a second languages. Rowley: Newbury House, 1997.

[2] Kintsch, W. and Van Dijk, Toward a model of discourse comprehension and production [J]. Psychological Review, 1978, (85): 363-394.

[3] He Xianyou. An Experimental Study on the Effect of Marking and the Mechanism of Machining [D]. South China Normal University, 1999.

[4] Lorch R F, Lorch E P, Imman W E. Effects of Signaling Topics Structure on Text Recall [J]. Journal of Educational Psychology, 1993, (2): 281-290.

[5] Bai Xuanjun, Zhou Yuanyuan.Experimental Research on the Influence of 
Training and Article Marking on the Content of Articles [J]. Journal of Tianjin Normal University (Natural Science Edition), 2004, (6): 68-71. 